\title{
Range and pattern of mandibular opening in patients with anterior displacement of temporomandibular disc without reduction
}

\author{
Monika Litko', Jacek Szkutnik', Marcin Berger', Magdalena Bakalczuk', Ingrid Różyło-Kalinowska² \\ ${ }^{1}$ Department of Functional Masticatory Disorders, Medical University, Lublin, Poland \\ ${ }^{2}$ Independent Unit of Propaedeutics of Dental and Maxillofacial Radiology, Lublin, Poland
}

Litko M, Szkutnik J, Berger M, Bakalczuk M, Różyło-Kalinowska I. Range and pattern of mandibular opening in patients with anterior displacement of temporomandibular disc without reduction. J Pre-Clin Clin Res. 2018; 12(2): 59-62. doi: 10.26444/jpccr/91610

\begin{abstract}
Introduction. Impairment of mandible mobility model is one of the criteria for clinical diagnosis of temporomandibular joint (TMJ) disc displacement.

Objectives. Analysis of the range and pattern of the mandibular opening in patients with anterior disc displacement without reduction.

Materials and method. According to the Research Diagnostic Criteria for Temporomandibular Disorders (RDC/TMD) the range and pattern of mandibular opening were analyzed in 49 patients ( 45 women and 4 men) with uni- and bilateral anterior disc displacement without reduction on MR imagines.

Results. The average range of opening in patients with unilateral lack of disc reduction was $33.74 \mathrm{~mm}$, and $28.93 \mathrm{~mm}$ in patients with bilateral lack of reduction. The difference between the average range of opening in both groups was statistically significant $(p<0.05)$. Uncorrected mandibular deviation was the most frequent opening pattern in patients with unilateral lack of reduction (19 out of 35 cases). In 11 out of 14 cases with bilateral lack of reduction the straight opening pattern was found.

Conclusions. TMJ disc displacement with bilateral lack of reduction during opening can cause greater opening limitation than unilateral lack of reduction. Anterior disc displacement without reduction in the MR images can clinically present with limitation of mandibular opening as well as correct range of this movement, which can cause difficulties in the clinical diagnosis of these problems.
\end{abstract}

\section{Key words}

temporomandibular joint disorders; temporomandibular joint disc; magnetic resonance imaging

\section{INTRODUCTION AND OBJECTIVE}

Clinical analysis of mandibular mobility model is a basic examination to asses the condition of the masticatory system, including function of the condyle-disc complex of the temporomandibular joint (TMJ). Analysis of free mandibular movements includes measurement of the range of movements, including range of mandibular opening as well as the assessment of the pattern of this movement. The range of the mandibular opening measured between the incisal edges of the upper and lower medial incisors according to different authors should be from 40 to $60 \mathrm{~mm}$, and have a straight pattern without lateral deviations $[1,2]$.

Limited range of mandibular opening below $35 \mathrm{~mm}$ and hard end-feel are one of the main criteria for identifying the anterior disc displacement without reduction [3].

Nevertheless, in many cases, precise diagnosis of intraarticular disorders can not be made solely on the basis of a clinical examination, because clinical methods for diagnosis of TMJ disorders, including the assessment of the disc position have certain documented limitations. Only the use of magnetic resonance (MR) images which is recognized as the gold standard in the diagnosis of intra-articular disorders

Addres for correspondence: M. Litko, Department of Functional Masticatory Disorders, Medical University, Karmelicka 7, 20-081 Lublin, Poland

Tel./fax +48 815287930

E-mail:monika.litko@umlub.pl

Received: 6 March 2018; accepted: 25 May 2018 allows for precise visualization and evaluation of the TMJ disc function in any position of the mandible without exposing the patient to the radiation [4].

According to the Research Diagnostic Criteria for Temporomandibular Disorders (RDC / TMD), clinical symptoms of anterior disc displacement in cases of unilateral lack of disc reduction with limitation of the mandible opening are accompanied by uncorrected mandibular deviation towards the joint in which the reduction does not occur $[3,5]$.

The literature rarely describes cases of disc displacement with bilateral lack of reduction [6]. We were not able to identify reports comparing of the range and pattern of mandibular opening in both types of these displacements.

The aim of this study was to analyse the range and pattern of the mandibular opening in patients with anterior disc displacement without reduction on one or both sides in the sagittal plane on MR imagines. A research hypothesis was formulated that the range of mandibular opening is decreased in people with bilateral lack of disc reduction compared to people with unilateral lack of reduction.

\section{MATERIALS AND METHODS}

MR images of TMJs were retrospectively analysed in patients seeking treatment for TMD, in whom TMJ disc displacement was suspected on the basis of the interview and clinical examination. MRI investigations of the TMJs were carried 
out in intercuspal position (IP) and open-mouth position (OMP), using silicon bite indices. All patients underwent bilateral MRI examinations of TMJs with a TMJ surfacecoil. MRI images were done with the aid of a 1.5-T MRI unit (Eclipse 1.5T; Picker). PD, T1, T2*-weighted fast spin-echo. MRI was performed in the oblique sagittal and the coronal projection.

Two experienced observers separately evaluated MR images. The observers were evaluated beforehand during a calibration session, at which interobserver reliability assessment revealed acceptable agreement $(\kappa=0.78)$. In cases of disagreement, the final assessment was made by consensus. The Bioethics Committee of the Medical University of Lublin, Poland approved this study (no. KE-0254/11/2015).

The MR images of 49 patients ( 45 women and 4 men) with uni- and bilateral anterior disc displacement without reduction were qualified for the presented study [7].

The age of participants ranged from 14 to 55 years. Patients with a history of facial trauma, systemic inflammatory arthritis, TMJ tumour or TMJ surgery were excluded from the study.

Afterward the range and pattern of the mandibular opening of selected patients were also analysed. The range of mandibular was measured in millimeters on the day of the MR examination using a ruler with a millimeter scale as the distance between the incisal edges of the upper and lower medial incisors corrected by the vertical incisal overlap. The deviation pattern was classified according to RDC/TMD as: - straight

- corrected deviation

- uncorrected deviation.

The obtained data were analysed using IBM SPSS Statistics Version 22 software. The inter-observer reliability of measurements was assessed using kappa statistic (Cohen's kappa, $\kappa)$. Student's t-test was used for uncorrelated data to test the significance of differences between means. The level of significance was set at $\mathrm{p}<0.05$.

\section{RESULTS}

The analysis of MR images of 49 patients revealed anterior disc displacement without reduction on one side in 35 cases and on both sides in 14 cases (Table 1, Figure 1 and 2).

Table 1. Temporomandibular disc displacement and range of mandibular opening

\begin{tabular}{lcccccccc}
\hline Anterior disc displacement & $\mathbf{n}$ & \multicolumn{6}{c}{ Range of mandibular opening $(\mathrm{mm})$} \\
\cline { 3 - 8 } & & $\mathrm{x}_{\min }$ & $\mathrm{x}_{\max }$ & $\overline{\mathrm{x}}$ & $\mathrm{SD}$ & $\mathrm{t}$ & $\mathrm{p}$ \\
\hline Without reduction on one side & 35 & 21 & 46 & 33.74 & 7.71 & 2,072 & $<0.05$ \\
\hline Without reduction on both sides & 14 & 18 & 36 & 28.93 & 6.29 & & \\
\hline
\end{tabular}

The average range of opening in patients with unilateral lack of disc reduction was $33.74 \mathrm{~mm}$ (minimum $21 \mathrm{~mm}$, maximum $46 \mathrm{~mm}$ ), and $28.93 \mathrm{~mm}$ (minimum $18 \mathrm{~mm}$, maximum $36 \mathrm{~mm}$ ) in patients with bilateral lack of reduction. The difference between the average range of opening in both groups was statistically significant (Table $1, \mathrm{p}<0.05$ ).

Uncorrected mandibular deviation was the most frequent opening pattern in patients with unilateral lack of reduction
Table 2. Temporomandibular disc displacement and pattern of mandibular opening

\begin{tabular}{lcccc}
\hline Anterior disc displacement & $\mathrm{n}$ & \multicolumn{3}{c}{ Pattern of opening } \\
\cline { 3 - 5 } & & straight & $\begin{array}{r}\text { corrected } \\
\text { deviation }\end{array}$ & $\begin{array}{r}\text { uncorrected } \\
\text { deviation }\end{array}$ \\
\hline Without reduction on one side & 35 & 5 & 11 & 19 \\
\hline Without reduction on both sides & 14 & 11 & 2 & 1 \\
\hline
\end{tabular}

(19 out of 35 cases). In 11 out of 14 cases with bilateral lack of reduction the straight opening pattern was found (Table 2).

\section{DISCUSSION}

Analysis of free mandibular movements, among them the measurement of the range of mandibular opening is the basic clinical examination in diagnosing of TMJ disc displacement. The clinical differentiation of disc displacement with and without reduction is based mainly on the occurrence or absence of reciprocal clicking and the accompanying specific disturbances of the mandibular mobility [4]. Definitely, the use of MR imaging, that allows the visualization of the articular disc, greatly expands the diagnostic possibilities of these disorders.

Literature data show that the agreement between clinical and MRI diagnosis of TMJ disc displacement varies from 59 to $90 \%$ and seems to result from the methodology and diagnostic criteria adopted in various studies $[4,8-10]$. The greatest non-compliance concerns the disc displacement without reduction in cases without restriction of the mandibular opening according to RDC/TMD. The conducted research confirmed that anterior disc displacement without reduction in the MR images can clinically present the limitation of the mandibular opening as well as the correct range of this movement, which causes difficulties in clinical diagnostics of these displacements (Table 1). In presented study the range of mandibular opening was 18 to $46 \mathrm{~mm}$, which also shows that there is a probability of making a false diagnosis of the type of disc displacement based on the limitation of the range of the opening.

Lack of disc reduction during opening may limit the range of this movement by mechanically blocking or even completely preventing translational movement in the affected joint [11]. In our study the smallest (only $18 \mathrm{~mm}$ ) range of mandibular opening was found in the group of patients with bilateral lack of disc reduction (Table 1). This range indicates that during opening only rotational movement occurs in the lower TMJ compartment. Additionally, in most cases with bilateral lack of reduction a significant limitation of the opening was accompanied by a straight opening pattern. Such a model of mandibular mobility without deviation probably results from the same type of disc displacement in both joints.

However, it is worth noting that the majority of patients with unilateral absence of reduction presented uncorrected mandibular deviation during opening and a statistically significantly larger mean range of this movement (Table 1). In these cases, greater opening range can be explained also by the possibility of compensating movement in the second joint in which the full disc reduction takes place, which is not possible in the patients with bilateral lack of reduction. 


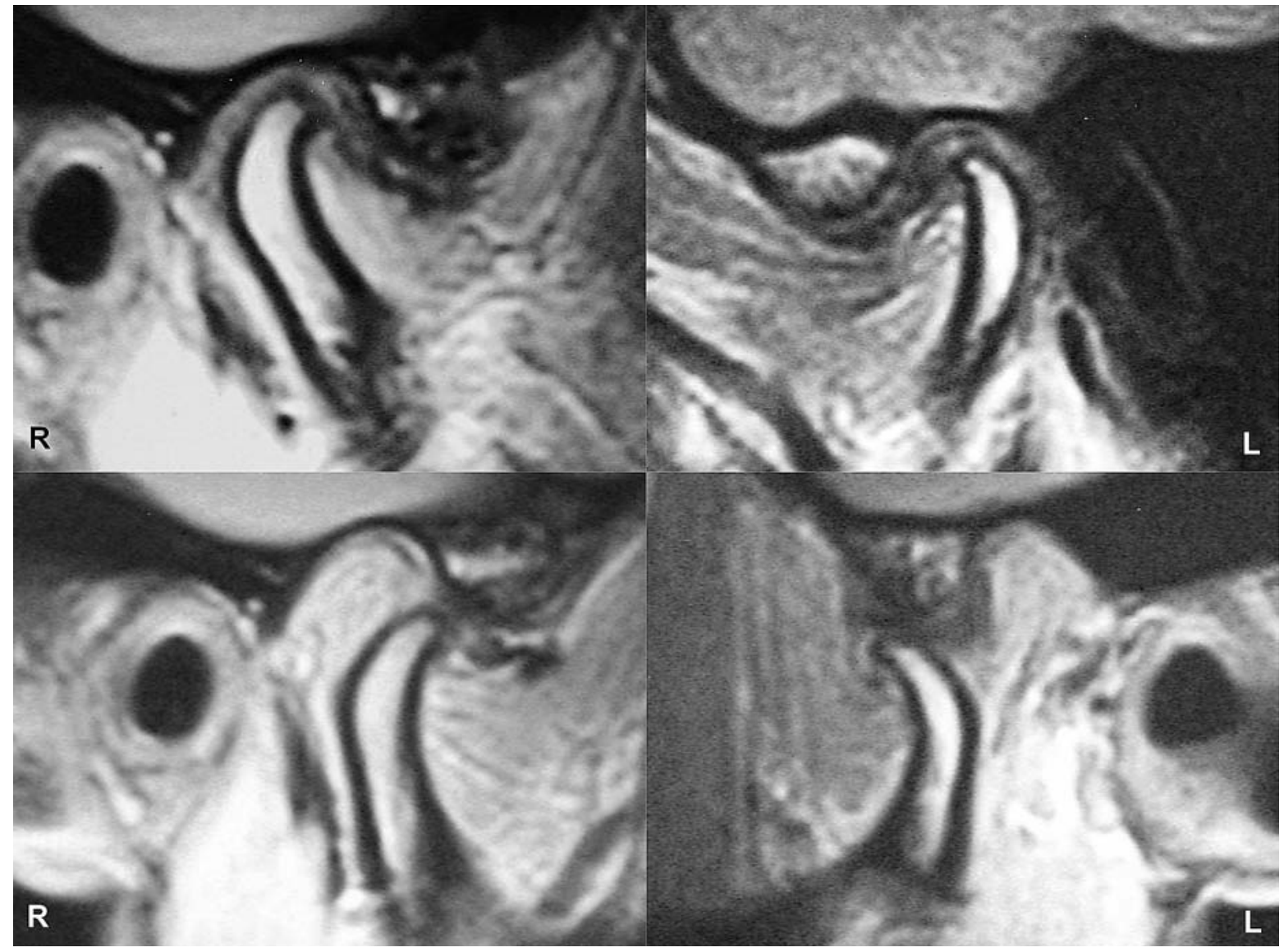

Figure 1. Bilateral anterior disc displacement in the sagittal plane without reduction in the right temporomandibular joint (above).

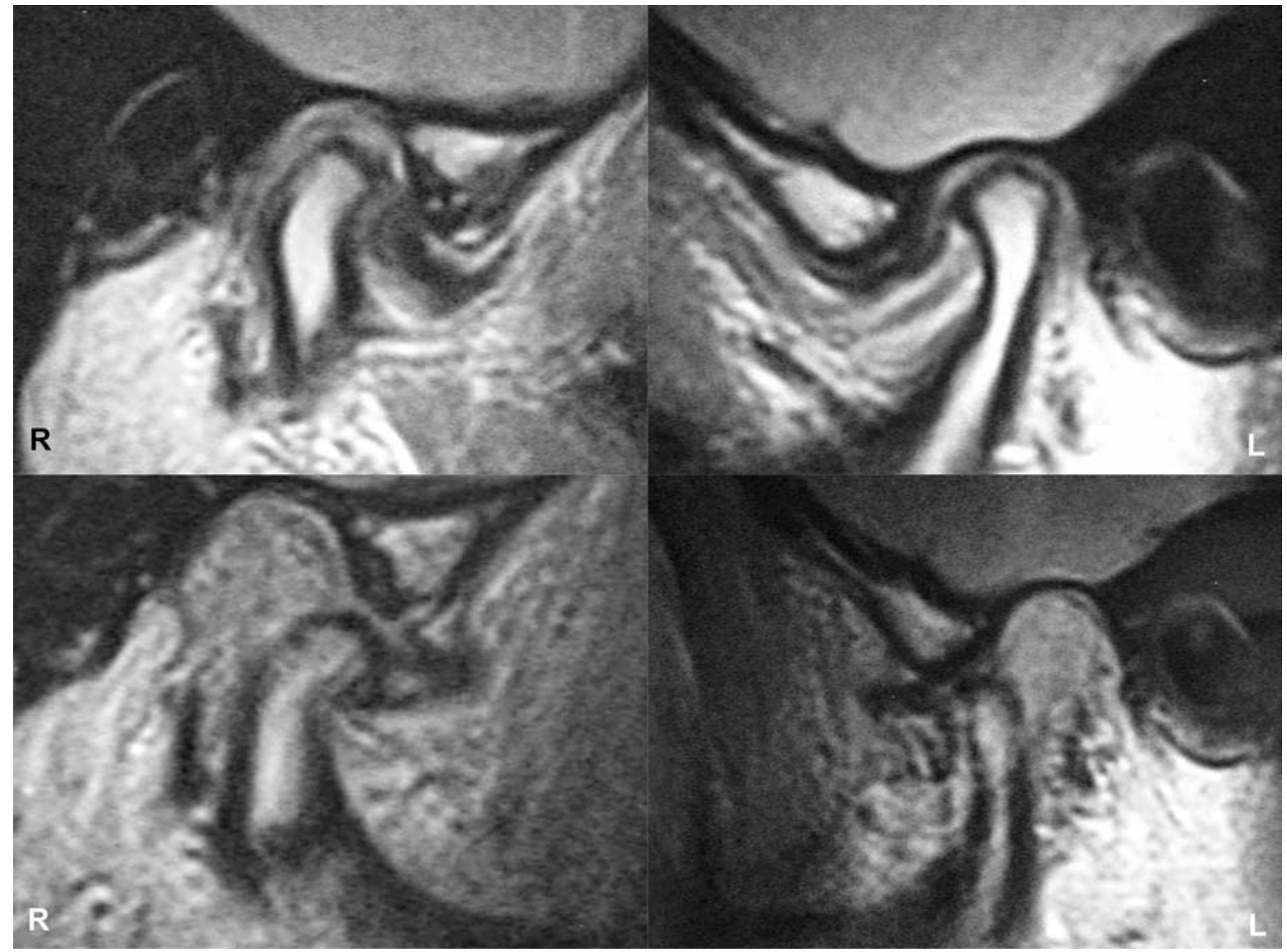

Figure 2. Bilateral anterior disc displacement in the sagittal plane without reduction in both temporomandibular joints (below). 
In the conducted studies, cases with a range of opening up to $46 \mathrm{~mm}$ occurred in the group of patients with unilateral lack of reduction, which is consistent with longitudinal studies describing the natural development of intra-articular disorders in cases with disc displacement without reduction. In patients who have not taken treatment the range of opening may progressively increase or even return to norm [12-15].

The presence of a proper range of opening in cases with chronic disc displacement without reduction may probably result from the following remodeling and adaptation of TMJ tissues, including changes in the shape of the articular disc, its elongation and loss of biconcave shape, as well as the elongation of the posterior attachment. These changes allow increasing the range of mandibular motion, and even in some patients, returning to the normal range of movements $[6,16-20]$.

The increase of the range of mandibular opening can be accompanied by changes in the opening pattern, such as appearance of corrected deviation or even straight path, which was observed in the examined patients (Table 2).

The presented research confirms that the clinical analysis of mandibular movements including the opening range and pattern can not be solely the basis for identifying the TMJ disc displacement without reduction and indicate the necessity (advisability) of conducting further research on the clinical symptoms of intra-articular disturbances in order to improve the diagnosis of these disorders.

\section{CONCLUSIONS}

TMJ disc displacement with bilateral lack of reduction during opening can cause greater opening limitation than unilateral lack of reduction. Anterior disc displacement without reduction in the MR images can clinically present with the limitation of the mandibular opening as well as with correct range of this movement, which can cause difficulties in the clinical diagnosis of these problems.

\section{REFERENCES}

1. Kleinrok M. Zaburzenia czynnościowe układu ruchowego narządu żucia. Lublin, Czelej, 2012.

2.Majewski S. W. Gnatofizjologia Stomatologiczna. Warszawa, PZWL, 2007.

3.Schiffman E, Ohrbach R, Truelove E, Look J, Anderson G, Goulet JP et al Diagnostic Criteria for Temporomandibular Disorders (DC/ TMD) for Clinical and Research Applications: recommendations of the International RDC/TMD Consortium Network and Orofacial Pain Special Interest Group. J Oral Facial Pain Headache. 2014; 28(1): 6-27.
4. Manfredini D, Guarda-Nardini L. Agreement between Research Diagnostic Criteria for Temporomandibular Disorders and magnetic resonance diagnoses of temporomandibular disc displacement in a patient population. Int J Oral Maxillofac Surg. 2008; 37(7): 612-6.

5. Osiewicz M, Lobbezoo F, Loster BW, Wilkosz M, Naeije M, Ohrbach R. Research Diagnostic Criteria for Temporomandibular Disorders (RDC/ TMD) - The Polish version of a dual-axis system for the diagnosis of TMD. RDC/TMD Form. J Stomatol. 2013; 1(66): 576-649.

6. Zhuo Z, Cai X. Results of radiological follow-up of untreated anterior disc displacement without reduction in adolescents. Br J Oral Maxillofac Surg. 2016; 54(2): 203-7.

7. Ahmad M, Hollender L, Anderson Q, Kartha K, Ohrbach R, Truelove EL et al. Research Diagnostic Criteria for Temporomandibular Disorders (RDC/TMD): Development of Image Analysis Criteria and Examiner Reliability for Image Analysis. Oral Surg Oral Med Oral Pathol Oral Radiol Endod. 2009;107(6): 844-60.

8. Park JW, Song HH, Roh HS, Kim YK, Lee JY. Correlation between clinical diagnosis based on RDC/TMD and MRI findings of TMJ internal derangement. Int J Oral Maxillofac Surg. 2012; 41(1): 103-8.

9. Kumar R, Pallagatti S, Sheikh S, Mittal A, Gupta D, Gupta S. Correlation Between Clinical Findings of Temporomandibular Disorders and MRI Characteristics of Disc Displacement. Open Dent J. 2015; 31(9): 273-81.

10. Kraus S, Prodoehl J. Disc displacement without reduction with limited opening: A clinical diagnostic accuracy study. Physiother Theory Pract. 2017; 33(3): 238-44.

11. Yang Z, Wang M, Ma Y, Lai Q, Tong D, Zhang F et al. Magnetic Resonance Imaging (MRI) Evaluation for Anterior Disc Displacement of the Temporomandibular Joint. Med Sci Monit Int Med J Exp Clin Res. 2017; 8(23): 712-8.

12. Manfredini D, Favero L, Gregorini G, Cocilovo F, Guarda-Nardini L. Natural course of temporomandibular disorders with low pain-related impairment: a 2-to-3-year follow-up study. J Oral Rehabil. 2013; 40(6): 436-42.

13. Sato S, Kawamura H, Nagasaka H, Motegi K. The natural course of anterior disc displacement without reduction in the temporomandibular joint: follow-up at 6, 12, and 18 months. J Oral Maxillofac Surg. 1997; 55(3): 234-8

14. Kurita K, Westesson PL, Yuasa H, Toyama M, Machida J, Ogi N. Natural course of untreated symptomatic temporomandibular joint disc displacement without reduction. J Dent Res. 1998; 77(2): 361-5.

15. Imirzalioglu P, Biler N, Agildere AM. Clinical and radiological followup results of patients with untreated TMJ closed lock. J Oral Rehabil. 2005; 32(5): 326-31.

16. Isberg A, Isacsson G. Tissue reactions associated with internal derangement of the temporomandibular joint. A radiographic, cryomorphologic, and histologic study. Acta Odontol Scand. 1986; 44(3): 160-4.

17. Panmekiate S, Petersson A, Akerman S. Some anatomical factors of the upper compartment of the temporomandibular joint related to the disc position. Int J Oral Maxillofac Surg. 1991; 20(6): 375-7.

18. Sato S, Sakamoto M, Kawamura H, Motegi K. Long-term changes in clinical signs and symptoms and disc position and morphology in patients with nonreducing disc displacement in the temporomandibular joint. J Oral Maxillofac Surg. 1999; 57(1): 23-9.

19. Cai X-Y, Jin J-M, Yang C. Changes in disc position, disc length, and condylar height in the temporomandibular joint with anterior disc displacement: a longitudinal retrospective magnetic resonance imaging study. J Oral Maxillofac Surg. 2011; 69(11): e340-6.

20. Hu YK, Yang C, Cai XY, Xie QY. Does condylar height decrease more in temporomandibular joint nonreducing disc displacement than reducing disc displacement?: A magnetic resonance imaging retrospective study. Medicine (Baltimore). 2016; 95(35): e4715. 